\title{
Responsabilidad Penal de las Personas Jurídicas: ¿Resulta el Compliance una medida suficiente y útil para el sistema judicial peruano?
}

José Ugaz SÁnchez-Moreno

Abogado por la Pontificia Universidad Católica del Perú. Estudios en el Institute of Social Studies, The Hague, Holanda. Especialización en Derechos Humanos por la Universidad de Salamanca.

Ex Procurador Ad-Hoc de la República.

Profesor Principal de Derecho Penal de la Pontificia Universidad Católica del Perú

José Domingo Pérez Gómez

Abogado por la Universidad Católica de Santa María. Máster en Derecho Constitucional por la Universidad Católica de Santa María. Máster en Derecho de la Empresa por la Universidad Católica de Santa María.

Máster en Gestión Pública por la Universidad ESAN.

Fiscal Provincial Titular del Primer Despacho de la Fiscalía Supraprovincial Especializada en Delitos de Corrupción de Funcionarios- Equipo Especial.

Marcos Tapia Rivas

Abogado por la Universidad San Martín de Porres. Máster en Derecho Penal por la Universidad Nacional Federico Villarreal. Doctor en Derecho por la Universidad Nacional Federico.

Profesor de Derecho Penal de la Universidad César Vallejo. Abogado Adscrito a la Procuraduría Pública Especializado en Tráfico llícito de Drogas del Ministerio del Interior.

\section{Richard Concepción Carhuancho}

Abogado por la Universidad San Martín de Porres. Máster en Derecho de Empresas por la Pontificia Universidad Católica del Perú.

Estudios de Doctorado por la Universidad Nacional Mayor de San Marcos. Juez Titular del Primer Juzgado de Investigación Preparatoria Nacional. 


\title{
RESUMEN:
}

El Compliance es un término bastante familiar entre las empresas norteamericanas e incluso en países de Europa. Sin embargo, es un concepto nuevo que se viene manejando en el Perú. La principal función del Compliance es garantizar la integridad de la compañía y para ello identifica riesgos y establece mecanismos que permitan que los mismos no se conviertan en un problema. Atendiendo a ello, ADVOCATUS tuvo la oportunidad de entrevistar a cuatro especialistas en la materia para que nos compartan sus reflexiones sobre Compliance en nuestro marco normativo, así como en el contexto social que nos encontramos.

\begin{abstract}
:
Compliance is a fairly familiar term among American companies and even in European countries. However, it is a new concept that that is being handled in Peru. The main function of Compliance is to guarantee the integrity of the company and for this it identifies risks and establishes mechanisms that allow them not to become a problem. In response to this, ADVOCATUS had the opportunity to interview four specialists in the field to share their reflections on Compliance in our regulatory framework, as well as in the social context we are in.
\end{abstract}

\section{1. ¿Cuál es el marco teórico que subyace a la responsabilidad penal de la persona jurídica?}

José Ugaz (JU): La Responsabilidad Penal de las Personas Jurídicas se introdujo mediante la Ley 30424 — vigente desde el primero de julio del 2017- Hasta antes de esa ley las personas jurídicas solo podían responder patrimonialmente como tercero civilmente responsable, en caso alguien de la empresa hubiera cometido un delito. A partir de esa fecha, primero para el delito de soborno trasnacional y luego se fue ampliando para otros delitos como lavado de activos y corrupción se asume que las personas jurídicas pueden ser involucradas en investigaciones penales como sujetos activos, como autores $y$, por lo tanto, ser pasibles de sanciones penales, al igual que las personas físicas.

José Pérez (JP): La responsabilidad penal de las personas jurídicas no es una novedad en el derecho penal peruano. El Código Penal incorporó sanciones de carácter penal o del proceso penal para las personas jurídicas a las cuales denominó "consecuencias accesorias". Esto ha sido una decisión político-criminal del legislador, porque procuraba establecer una respuesta punitiva frente a aquellos delitos donde se encontraba vinculada o involucrada la persona jurídica por la comisión del hecho punible por parte de su directivo, administrador o representante.
Es por eso que el artículo 105 del Código Penal regula las consecuencias accesorias de la persona jurídica. Esta es una suerte de responsabilidad subsidiaria - que estaba definida en ese entonces - para el caso que la persona natural o física, como ya lo había indicado, el representante o quien actúa en nombre de la persona jurídica cometa los delitos. Es por eso que en su oportunidad se estableció en el Acuerdo Plenario 7-2009, que no eran una especie de penas accesorias, como vendría a ser la inhabilitación, sino un complemento dependiente o accesorio de una pena principal que se le deba imponer al autor del delito.

Por ello que se hace referencia de que tienen una condición accesoria, vicaria, o paralela a la ley. Es decir, que es una condición exigida de la ley, una exigencia normativa que, para su imposición, se debía identificar la comisión del hecho por parte de la persona natural como autora del hecho punible, y luego conectar esta responsabilidad a la persona jurídica.

La pregunta es, ¿Cuál es la base teórica actualmente?, pues más allá de esta pequeña introducción, la Ley 30424 es la que finalmente establece la responsabilidad administrativa de las personas jurídicas, Ley del 21 de abril de 2016; el Decreto Legislativo 1352, que amplía el tema de la responsabilidad administrativa de las personas jurídicas, del 06 de enero de 2017; la Ley 30835, que modifica la Ley 30424, 
que va regulando esta responsabilidad administrativa; el Reglamento publicado mediante Decreto Supremo 02-2019, del 09 de enero de 2019, que establece los componentes de un modelo de prevención de delitos, mecanismos necesarios para que los fiscales puedan investigar y solicitar sanciones en estas medidas administrativas contra las personas jurídicas, el marco legal para la determinación de la persona jurídica en caso se solicite algún tipo de eximente de responsabilidad administrativa o atenuación de la misma.

Toda esta base legal viene actualmente regulando la responsabilidad administrativa de las personas jurídicas. En preciso señalar que, no es que se adopte un tipo de responsabilidad penal, sino responsabilidad administrativa derivada de la comisión de un delito, que tiene consecuencias netamente administrativas.

Esto no es una novedad, definitivamente en nuestra legislación ha tenido determinadas denominaciones, como lo he indicado. Si debe establecerse la comisión de un delito, ahora se puede solicitar la sanción por la comisión del hecho a la persona jurídica.

Asimismo, quiero hacer mención a lo que César San Martín señaló en una conferencia del 30 de mayo de 2019, a propósito de la pregunta. Él ha indicado que, sea se denomine responsabilidad administrativa o penal, es un "tema de etiquetas". Él cree que debe ser denominada responsabilidad administrativa y no penal; sin embargo, al ser un tema de etiquetas, sea administrativa o sea penal, finalmente son los criterios de atribución, en ambos casos, iguales.

Si es un tema denominado administrativo, es porque tenemos un antecedente italiano $o$ alemán, y si lo considera penal, es porque es un tema francés, español o argentino.

Marcos Tapia (MT): El pilar fundamental de cualquier investigación, análisis, experimento o propuesta de desarrollo de un trabajo resulta ser el marco teórico. El marco teórico de la responsabilidad penal de las personas jurídicas, tiene que ver con el objeto de la Ley 30424 — Ley que regula la responsabilidad administrativa de las personas jurídicas por el delito de cohecho activo transnacional; ampliado a los delitos de corrupción, lavado de activos y financiamiento del terrorismo-, y su modificación, por medio del Decreto Legislativo 1352. Ambas son formas de asegurar que se aplique la responsabilidad penal, civil o administrativa a las personas jurídicas involucradas en los delitos en mención, en consonancia con las políticas del Estado en materia de lucha contra la corrupción.

Richard Concepción (RC): La responsabilidad de las personas jurídicas comprende dos áreas plenamente diferenciadas, la responsabilidad administrativa y la responsabilidad penal de la persona jurídica.

Por un lado, la responsabilidad administrativa de la personas jurídica se encuentra regulada en la Ley 30424 , con su modificatoria introducida por el Decreto Legislativo 1362, la cual se aplica a una pluralidad de delitos, entre ellos, a los delitos de cohecho internacional, lavado de activos, terrorismo, y minería ilegal, en la medida que hayan sido cometidos a nombre de la persona jurídica y en beneficio de la misma, en cuyo caso podría imponérsele medidas administrativas como multa, inhabilitación, cancelación de derechos reconocidos a su favor, clausura de locales y la disolución.

Por el otro lado, tenemos la responsabilidad penal de las personas jurídicas. La misma se encuentra regulada en el Código Penal y en el Código Procesal Penal, para lo cual se exige su incorporación formal al proceso penal, en cuyo caso se requerirá que se identifique a la persona jurídica, se precise la cadena de atribución existente en su contra y la fundamentación legal correspondiente, a fin de que el seno del proceso penal puede defenderse de los cargos que se le atribuyen, para finalmente decidirse si es viable o no la imposición de una consecuencia accesoria en contra de la persona jurídica, como la multa, dictado de prohibiciones, suspensión de actividades, disolución y liquidación de la sociedad y clausura de locales o establecimientos - siendo una suerte de sanción especial a la persona jurídica-.

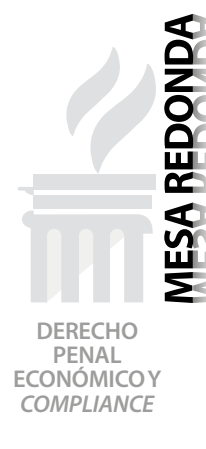


2. ¿Qué es un programa de cumplimiento normativo o Compliance penal? Y, ¿Es suficiente con la tenencia, diseño y/o implementación de un programa de cumplimiento para que se atenúe o exima de responsabilidad penal a la empresa?

JU: Los programas de cumplimiento son modelos que se implementan al interior de las empresas para evitar riesgos que puedan terminar en la comisión de actos delictivos. Esos programas de cumplimiento tienen ciertos estándares universales en estos tiempos e incluso pueden ser objeto de certificación; por ejemplo, para obtener el ISO 37001 que es el ISO antisoborno. Sin embargo, de acuerdo a la Ley y su Reglamento, no vas a tener el programa de cumplimiento para ser exonerado o atenuada la responsabilidad penal; sino que se tiene que hacer un programa de primer nivel que sea consistente y cuente con una opinión favorable de la Superintendencia de Mercado de Valores -en adelante, la "SMV"-, que es el ente supervisor que ha establecido la Ley.

JP: Compliance es un anglicismo, que en español vendría a ser "cumplimiento a la norma" —un modelo de cumplimiento, técnicamente nuestra legislación nos indica "prevención"- y en buena cuenta se define como la regulación interna que debe adoptar la persona jurídica en su organización, con el fin de garantizar la ética empresarial y prevenir la comisión de actos delictivos.

Un programa de cumplimiento lo que pretende es afianzar o identificar los valores éticos de la empresa para el desarrollo de sus procesos, para la toma de decisiones. Técnicamente es un elemento legal, es el marco legal que ha impuesto el legislador para el desarrollo empresarial. En ese sentido, hay que tomar en cuenta que es una intervención del estado en la esfera privada porque para la responsabilidad administrativa, acordémonos ha establecido como sanciones: la multa, la inhabilitación, la disolución y la intervención. Asimismo, al establecerse un modelo de cumplimiento o prevención como una exigencia empresarial lo que está indicándonos el poder estatal es que, el privado, dentro de su autonomía tiene que establecer cuál es el mejor modelo para determinar la naturaleza de la actividad que realiza, los riesgos en los cuales pueden darse la comisión de hechos con contenido penal, la necesidad de prevenir u organizarse en cumplimiento de la norma y cuáles van a ser las mejores medidas de vigilancia y autocontrol que puedan tener.

Un modelo de prevención, definitivamente a la pregunta que me realiza, no va a suponer definitivamente que no vaya a cometerse ningún tipo de hecho con contenido penal por parte de los trabajadores o de alguno de los directivos. Definitivamente sí va a ayudar a que todos los niveles empresariales adopten las decisiones más correctas; sin embargo, resalto no podría garantizarse que no podría haber algún tipo de actuación indebida por dentro de la autonomía de una persona que forma parte de la empresa, debido a que podría incumplir el modelo de prevención.

Ahora el modelo de prevención o de cumplimiento definitivamente tiene que cumplir con las necesidades o el modelo que ha adoptado la organización, no es lo mismo una empresa grande que una empresa pequeña, no es lo mismo el modelo de prevención, de cumplimiento que supone realizar una auditoria interna para determinar cuál es el proceso o los procesos que realiza la empresa que, simplemente, adoptar un modelo sin haber realizado el análisis o el estudio de las actividades que realiza.

MT: El Compliance o programa de cumplimiento normativo es un conjunto de reglas y procedimientos que la persona jurídica adopta e implementa en su organización como modelo de prevención. Este modelo es adecuado a su naturaleza, riesgos, necesidades y características, y consiste en medidas de vigilancia y control idóneas para prevenir los delitos en mención o para reducir significativamente el riesgo de su comisión - esto de conformidad a lo señalado por artículo 17 de la Ley-.

No es suficiente la tenencia de este programa de cumplimiento normativo ya que, de ser el 
caso, este debe ser supervisado por un tercero - tal cual lo menciona el artículo 19 de la Ley-. Siguiendo este orden de ideas, el plan debe contener lo siguiente:
a) Mapa de riesgos
b) Manual de cumplimiento
c) Revisión operativa
d) Información
e) Auditorias de cumplimiento
f) Cultura de transparencia
g) Revisiones periódicas
h) Canal de denuncias
i) Procedimientos de investigación
j) Código de ética ${ }^{1}$

$\underline{\mathrm{RC}}$ : El programa de cumplimiento que se implemente al interior de una empresa puede ser de dos tipos:

1) El programa de cumplimiento efectivo apunta al cumplimiento efectivo del Derecho, de tal suerte que la propia estructura organizativa de la empresa deberá implementar un programa encaminado a evitar la comisión de delitos.

2) El Compliance penal exige la identificación de riesgos penalmente relevantes para la empresa, el diseño de reglas específicas para hacerlas frente y la aplicación efectiva de las mismas.

Es claro que la tenencia o implementación de un programa de cumplimiento servirá para establecer el grado de responsabilidad administrativa de la persona jurídica de la empresa frente a la comisión de delitos, sea para eximirlo o atenuarle su responsabilidad frente a la concretización de riesgos de carácter penal, así tenemos que:

a) En el caso que la empresa haya cometido delito y se acoge a un programa de cumplimiento, ello servirá para atenuar su responsabilidad. b) Distinto es el caso, en donde la empresa de manera antelada se haya acogido a un programa de cumplimiento de vigilancia y control para prevenir la comisión de delitos, en cuyo caso, la persona jurídica estará exenta de responsabilidad.

3. ¿El Compliance o programa de cumplimiento normativo aplicable en las empresas, contribuye a combatir la criminalidad?

JU: Es un elemento preventivo más, no es una vacuna definitiva. Sin embargo, no hay duda alguna que, disminuye o elimina en algunos casos los riesgos, pero lo importante no es tanto que se tenga formalmente un programa, sino que haya un cambio de cultura en los niveles más altos directivos y gerenciales de la empresa para que efectivamente se apliquen las mejores prácticas de gobierno corporativo y se reduzcan los espacios que puedan dar pie a que se cometan actos de corrupción, delitos, fraudes $u$ otras irregularidades.

JP: Ciertamente sí, porque parte de la política criminal del Estado y en cumplimiento de la legalidad, el Estado establece mecanismos para poder reducir o adelantarse a la comisión de hechos con contenido penal como son el lavado de activos, crímenes de corrupción, para el tema también de financiamiento del terrorismo -este último no es el tema al que me aboco, sino al lavado de activos y corrupción de funcionarios-.

Obviamente, el Estado lo realiza siguiendo recomendaciones de las organizaciones internacionales que ven por conveniente establecer obligaciones a la organización privada de las personas jurídicas, por eso no es extraño si seguimos esa tendencia mundial de que el Estado ingrese a la esfera privada a través de este modelo para prevenir esta comisión de hechos, sobre todo porque en una sociedad o en un mercado actual en donde el Estado contrata

1. Cfr. Economistas Reigosa \& Varela, España. 
con el privado, continuamente el Estado tiene que buscar estos mecanismos para prevenir la comisión de hechos delictivos más que buscar simplemente sancionarlos.

Es por eso que, en el derecho comparado, podemos establecer diversas modalidades de programas de cumplimiento que son de vital importancia, precisamente, para los grupos empresariales porque es una manera de autorregularse y esta manera de autorregularse para lo que permite ayudar, es que es una contribución del privado a la actividad pública, porque el privado está asumiendo este compromiso de lucha contra la corrupción, prevención de actividades de lavado de activos y financiamiento del terrorismo y de esta manera se suma a políticas públicas que son estas que te digo: la lucha contra la corrupción, la impunidad de lavado de activos y el financiamiento de terrorismo.

MT: Teniendo en cuenta que la criminalidad organizada - Ley 30077- tiene un ámbito de acción mucho mayor a lo que pretende la Ley 30424 - y su modificatoria, Decreto Legislativo 1352 - considero que regular la responsabilidad administrativa de las personas jurídicas involucradas en los delitos en mención, solo es un paliativo en la lucha contra la criminalidad organizada que abarca un número mayor de tipos penales —artículo 3 de la Ley 30077-, siendo este programa estrictamente preventivo en salvaguardar el patrimonio empresarial particular o estatal.

RC: El programa de cumplimiento normativo contribuye a combatir la criminalidad, en la medida que la persona jurídica cuente con un modelo preventivo frente a los riesgos jurídicos penales relevantes que se presenten como consecuencia de su desempeño de actividades comerciales, entre ellos tenemos:

1) La existencia de un órgano de la empresa que se encargue de la función de auditoría interna de prevención, debiendo contar para tal efecto, con recursos humanos, logística y prerrogativas funcionales para cumplir dicha función.
2) Diseño y aplicación de medidas encaminadas a la identificación de las actividades que generen riesgos en la comisión de delitos, para lo cual se requerirá contar con:

i) programas que prevengan la comisión de delitos;

ii) programas que eviten la utilización de la empresa para la comisión de delitos;

iii) la existencia de sistemas de denuncia y recepción de información sobre la comisión de delitos, con el carácter de reservado y protección de datos del denunciante.

3) Supervisión interna sobre la aplicación del programa de cumplimiento normativo a la persona jurídica, con el objeto de identificar sus fallas, y con ello prevenir de manera eficiente la comisión de delitos futuros.

\section{4. ¿Cuáles son los efectos del Compliance en el derecho penal sustantivo y en ámbito del derecho procesal penal?}

JU: En el ámbito del Derecho Procesal Penal tienes por mandato de la Ley la posibilidad de ser una causa atenuante o eximente de responsabilidad penal; siempre y cuando tenga una opinión favorable para esos efectos y tenga una evaluación positiva de la SMV.

Desde el punto de vista del Derecho Penal sustantivo, es un elemento modificador de la responsabilidad penal, en la medida que se puede haber cometido el delito del uso de la empresa; no obstante, si la empresa contaba con un modelo consistente y fue burlada por las personas físicas que cometieron los delitos, entonces eso tiene un impacto sobre la responsabilidad penal de la persona jurídica.

JP: Esto está establecido en la Ley 30424, una persona jurídica que tenga un modelo adecuado de un modelo de prevención, de un programa de cumplimiento antes de la comisión de delitos que señala la norma: de corrupción de funcionarios, de lavado de activos, de financia- 
miento de terrorismo, puede ser eximida la responsabilidad administrativa; es decir, no habrá multa, no habrá inhabilitación y no habrá las otras medidas que debería aplicarse a la persona jurídica. Ahora si es que este modelo de prevención no es adecuado, pero se tuvo antes de la comisión del hecho delictivo; la Ley 30424 señala que la responsabilidad puede ser atenuada, entonces ya vemos una cierta injerencia dentro del derecho penal.

Ahora, ¿Quién determina que este modelo sea el adecuado? Según la Ley, la Superintendencia del Mercado de Valores, es lo que señalaba la Ley. Y, se tendría que establecer en base a varios indicadores: análisis y corrección de los riesgos; compromiso de los órganos de gobierno; autonomía y recursos; políticas y procedimientos; evaluación de riesgos; etcétera. Pero, es ahí donde entramos al tema del Derecho Procesal Penal; porque, definitivamente, si es que se inicia una investigación y el fiscal recibe la denuncia y cita a los representantes de la persona jurídica y estos, en el desarrollo de la investigación, demuestran de que tenían un programa de prevención adecuado; entonces, el fiscal no tendría por qué proseguir con el proceso respecto a la persona jurídica.

Seguidamente, ¿Cómo el fiscal determinaría que este es un programa de cumplimiento o programa de prevención adecuado? Lo que ya dice la Ley 30424; es decir, a través del informe que tendría que dar la Superintendencia de Mercados de Valores. Es decir, se introduce ese informe como una pericia institucional. ¿Y qué es una pericia institucional? La pericia que se actúa fuera del proceso. Es decir, la que te proviene de un ente público. En ese sentido, si el fiscal tiene ese elemento de corroboración o de investigación y de convicción para determinar de que la persona jurídica tenía un modelo de prevención adecuado, no tendría por qué continuar con la causa y, por lo tanto, podría archivar respecto a la persona jurídica. Eso es más o menos la intervención que tiene el Programa de Cumplimiento y de Prevención en estos dos aspectos.

MT: Considero que tiene un efecto mínimo en el derecho penal, en razón que la Ley solo se cir- cunscribe a cuatro tipos penales - cohecho activo transnacional, corrupción, lavado de activos y financiamiento del terrorismo-. De acuerdo a la estadística de criminalidad, las personas jurídicas tienen un mínimo de participación en comparación con la responsabilidad personal, pero cuando se debe aplicar como medida administrativa desde una multa hasta llegar a una posible disolución o uso de las medidas cautelares, entonces esta medida resulta acertada.

En el derecho procesal penal, por tener directa relación con la norma sustantiva, sus efectos deben ir de la mano entre ambos. El derecho penal sustantivo describe el precepto prohibitivo con su respectiva sanción, por lo que el derecho procesal regula las formas de aplicación, en cuanto a la investigación y al procesamiento, teniendo en cuenta que el Código Procesal Penal regula a las personas jurídicas desde el artículo 90 al artículo 93.

RC: El Compliance criminal debería tener incidencia en el campo del derecho penal y en el campo del proceso penal, a propósito de incorporación de una persona jurídica al proceso penal y al momento de decidirse la imposición o no de consecuencias accesorias a la persona jurídica — suerte de sanción a las personas jurídicas-, por lo siguiente:

1) En cuanto al tópico de la incorporación de las personas jurídicas al proceso penal, encaminado al emplazamiento de la persona jurídica, a efectos que dentro del marco del proceso penal pueda defenderse de la cadena de atribución que se ha formulado en su contra - tema procesal-, existe controversia respecto a los presupuestos procesales exigidos para ello:

i) Una primera postura jurisprudencial sostiene que debe evaluarse el pedido de incorporación de la persona jurídica al proceso penal, verificándose la identificación de la persona jurídica, la existencia de una cadena de atribución - hechos que relacionen a la persona jurídica con los delitos investigados $-y$ la fundamentación legal correspondien-

(n) 
te; sin ingresar al análisis sobre la relevancia penal o no de la cadena de atribución - soporte probatorio o no de la cadena de atribución - por tratarse de asuntos que deberán ventilarse al interior del proceso penal, se entiende a través de los mecanismos procesales que correspondan;

ii) Una segunda postura jurisprudencial que exige que los hechos imputados en contra de la persona jurídica hayan sido señalados previamente en la Disposición de Formalización y Continuación de la Investigación Preparatoria, postura bastante discutible, por los siguiente: i) establece un requisito procesal no previsto en el propio artículo 91 del Código Procesal Penal; ii) se entiende que la Disposición de Formalización y Continuación de la Investigación Preparatoria es para imputar cargos en contra de la persona natural como imputado, y no contra la persona jurídica, que tiene su propio mecanismo procesal de imputación, a saber el requerimiento de incorporación de la persona jurídica y la resolución judicial constitutiva que lo aprueba - tal como lo sostiene Caro Coria-; iii) exigir que la imputación en contra de la persona jurídica conste en la Disposición de Formalización y Continuación de la Investigación Preparatoria haría innecesario transitar por el mecanismo procesal de la incorporación de la persona jurídica al proceso penal.

iii) Una tercera postura planteada por Caro Coria, según el cual para decidir la incorporación de una persona jurídica al proceso penal, además de cumplirse los requisitos establecidos en el Acuerdo Plenario 7-2009, debe analizarse la existencia de defectos en la organización o la deficiente administración de riesgos, en cuyo caso, importará verificar una hipótesis de no implementación de programas de Compliance para prevenir delitos, de donde se sigue que la tenencia o no de un programa de Compliance criminal será un requisito a verificar para decidir la incorporación de una persona jurídica al proceso penal.

2) En lo que toca a la imposición de consecuencias accesorias en contra de una persona jurídica dentro del marco de un proceso penal - tema de derecho penal sustantivo-, del mismo modo, importará si la persona jurídica se acoge o no a un programa de Compliance criminal, pues ello podrá invocarse para eximirle o atenuarle su responsabilidad, de cara a la adopción de consecuencias accesorias en su contra.

5. ¿Considera que es importante que los estudios jurídicos implementen un área dedicada al Compliance penal para prevenir potenciales actos de corrupción?

JU: Los estudios jurídicos tomaron con mucho entusiasmo la vigencia de la norma; algunos de ellos incluso ampliaron sus áreas de prestación de servicios, contrataron profesionales, pero luego se ha quedado a determinar la realidad de que los abogados no necesariamente son los primeros llamados a trabajar en programas de Compliance. Esto tiene que ver, más que con temas jurídicos — sin dejar de mencionar que tiene que ver con temas jurídicos-, pero en primer orden tiene que ver más con sistemas de riesgos, monitoreo de procesos, análisis de sistemas $y$, por lo tanto, requiere de un profesional de otro perfil.

Los profesionales del Derecho somos complementarios al trabajo que hacen, a quienes diseñan los procesos de Compliance y, establecen las áreas de riesgo de las empresas.

JP: La pregunta es bastante compleja. Si es que debería tener dentro de su área, para brindar ese servicio, va a depender del Estudio, a qué actividad se dedica. Si ofrece un servicio corporativo, entiendo que parte de ello va a ser el ofrecimiento de un programa de cumplimiento.

Ahora, si la preguntas que los Estudios Jurídicos, como empresas, deberían tener también 
un programa de prevención o un programa de cumplimiento para el propio estudio o como una entidad privada; entonces un claro ejemplo que yo te podría decir, sin antes primero mencionar y recalcar que la respuesta es "sí". Yo creo que sí, es necesario.

En seguida, te tendría que poner el claro ejemplo de lo que fue la investigación en el caso OreIlana; es decir, estábamos hablando de un estudio de abogados que supuestamente brindaba servicios, pero que ya incurrió en la comisión de hechos delictivos. Entonces, un programa de cumplimiento o de prevención, entiendo de que pudo haber advertido o puede advertir en condicional o a futuro, no solamente sometiéndome a este caso, podría sí ayudar a un estudio jurídico a orientar adecuadamente los fines de su organización.

MT: Creo que sí, ya que la obligación de la implementación de este programa de cumplimiento normativo es para las personas jurídicas que generen bienes y servicios que podrían involucrarse en actos de criminalidad vinculados en mayor escala a actos de corrupción y de lavado de activos, dejando abierta la posibilidad en el marco normativo de que podrían ser todo tipo de personas jurídicas, sean particulares, estatales, reguladas por el Código Civil o por la Ley General de Sociedades, entre otros.

Es preciso indicar que, no se encuentra nada en la normativa vigente que impida a los estudios jurídicos ofrecer los paquetes de implementación de cumplimiento normativo a través de asesorías.

RC: Considero que sí es importante que los estudios jurídicos cuenten con un área dedicada a prevenir potenciales actos de corrupción. Esto claro está, en la medida que exista un ente regulador que los fiscalice, en cuanto al cabal cumplimiento de los programas de Compliance criminal en las empresas; de lo contrario, se podría llegar al escenario - que nadie desea- de que el acogimiento formal a dichos programas constituya una licencia para cometer delitos, bajo el entendido que su simple acogimiento signifique que no se active consecuencia jurídica alguna en su contra.

6. En el supuesto de una pequeña empresa y partiendo del elevado costo de la implementación de un programa de cumplimiento ¿Qué tanto puede compensar la implementación de dicho programa preventivo?

JU: Las pequeñas empresas, efectivamente, no tienen la capacidad de costear procesos de Compliance muy costosos, pero como su estructura corporativa también es bastante más simple se pueden hacer algunos ajustes fundamentales como: contar con un Código de Ética, entrenamiento mínimo para el personal, contar con algún sistema de captación de denuncias anónimas sobre inconducta funcional y, sí básicamente, los niveles más simples del Programa de Cumplimiento y eso, en realidad, lo podrían obtener con una asesoría mínima y que no fuera muy costosa. En la actualidad, incluso pueden adoptar sistemas de cumplimiento sin la necesaria intervención de un profesional, por ejemplo, mirando ahora a través del internet otros modelos de empresas de similar tamaño y estructura.

JP: Lo que sucede es que las pequeñas empresas, cuando tengan vocación de crecer, definitivamente van a tener que adoptar modelos de prevención o cumplimiento. Es voluntario.

En este orden de ideas, si es que la proyección de la pequeña empresa es crecer dentro del mercado, debe saber que va a estar sometido a de que sus funcionarios, sus trabajadores, sus directivos puedan tener algún tipo de vinculación con el aparato público. Y en ese orden de ideas, de no tener un programa de cumplimiento, podría verse implicado o envuelto, la empresa, en algún tipo de responsabilidad administrativa por la comisión de alguno de sus trabajadores.

Claro está, y volvemos a una de las respuestas que di anteriormente, va a depender mucho del objeto de la empresa, el tamaño de la empresa. No es lo mismo tener un programa de

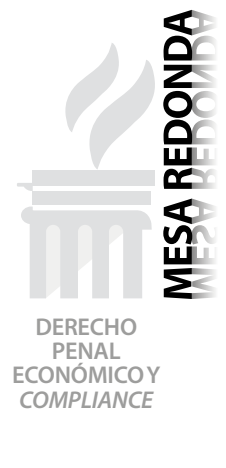


cumplimiento para una pequeña empresa que probablemente para una gran empresa. No es lo mismo realizar o adoptar un programa de cumplimiento luego de una auditoría que haya podido tener la empresa e identificar cuáles son los procesos y cuáles son las deficiencias que puedan mejorarse o cambiarse; que aquella que decide adoptarlo o adquirirlo. Entonces, ¿es necesario? Considero que sí. Ayuda a la prevención, permite la evaluación de riesgos y, definitivamente, si la empresa busca crecer, lo va a tener que adoptar.

MT: Una de las finalidades de esta Ley es la prevención de la comisión de actos ilícitos - ya identificados- donde la persona jurídica se pueda ver involucrada. Por medio de los programas de cumplimiento se trata de evitar recibir sanciones de carácter administrativo y/o penal, en razón de que la escala de sanción va desde una multa hasta la disolución de la persona jurídica.

La implementación de este programa a través de una certificación definitivamente debe tener un costo, y considero que este costo también dependerá de la magnitud y envergadura de la persona jurídica, pero la Ley — tal cual lo indica el artículo 19 de la misma - señala que el modelo de prevención puede ser certificado por terceros debidamente registrados y acreditados — como el caso de la norma ISO_, por lo cual esta certificación es opcional, ya que la legislación no obliga a la misma.

RC: Resulta claro que implementar un programa de Compliance criminal significa incrementar significativamente los costos de operatividad de las personas jurídicas, determinando que sea inaccesible para las pequeñas empresas.

Sin embargo, cabe señalar que su adopción debe verse como una inversión a largo plazo para las pequeñas empresas, en razón a que:

i) se les incentivará a internalizar una cultura de respeto al Derecho y de prevención de riesgos de carácter penal;

ii) el entendimiento que la comisión de delitos en el seno de la empresa -riesgo propio de toda empresa-, podría significar su liquidación, en cuanto a la reputación de la empresa en el mercado, y a la aplicación de consecuencias jurídicas negativas en su contra -intervención de la empresa e incautación de su patrimonio-. 